\title{
NEUTROPHIL GELATINASE-ASSOCIATED LIPOCALIN (NGAL) AS AN EARLY BIOMARKER OF ACUTE KIDNEY INJURY IN HEPATIC PATIENTS
}

\author{
Yousef Ahmed Kadry ${ }^{1}$,Abd Allah Abd El Aziz Abd Allah ${ }^{1}$, Atef Abd El Aziz Abd El Hadi Ali and Hisham \\ Mohammed Omar'. \\ ${ }^{1}$ Internal Medicine Department, Faculty of Medicine, Zagazig University, Egypt. \\ ${ }^{2}$ Clinical Pathology Department, Faculty of Medicine, Zagazig University, Egypt.
}

\begin{abstract}
Background :Acute kidney injury(AKI) is common in cirrhosis but differential diagnosis remains a challenge. Serum creatinine ( $\mathrm{SCr}$ ) less sensitive in reflecting renal dysfunction in cirrhotic patients. Aim of the Work: to study the usefulness of NGAL as an early biomarker of AKI in cirrhotic patients. Subjects and Methods: 80 subjects included, classified into 3 groups: GroupI: 10 control subjects. Group $\Pi(\mathrm{Gp} \Pi)$ : 40 compensated hepatic patients without AKI, further subdivided into four subcategories: (GpПa1): 10 patients with $\mathrm{HCV}$ under interferon plus ribavirin therapy. (GpПa2): 10 patients with $\mathrm{HCV}$ not under interferon or ribavirin therapy. (GpПb): 10 patients with Bilharzial liver fibrosis. $(\mathrm{Gp} \Pi \mathrm{c})$ : 10 patients with combined HCV and Bilharzial liver fibrosis. Group Ш (GpW): 30 decompensated hepatic patients with AKI, further subdivided into three subcategories: (Gp Шa): 10 patients with Acute tubular necrosis . (Gp Шb): 10 patients with hepatorenal syndrome . (Gp Шc): 10 patients with Pre-renal azotemia. All participants were subjected to the routine lab investigations in addition to specific lab test plasma NGAL (pNGAL).Results: No significant difference was found in kidney function parameters (SCr, urea, GFR) between patients with AKI and patients without AKI. However, patients with AKI had higher pNGAL compared to patients without AKI. There were significant difference among group III subcategories, patients with ATN had pNGAL levels markedly higher (mean $295 \mathrm{ng} / \mathrm{ml}$ ) compared to those of patients with PRA (mean $86.5 \mathrm{ng} / \mathrm{ml}$ ), Patients with HRS had intermediate values \{mean $142 \mathrm{ng} / \mathrm{ml}$ \}. In patients with ATN, pNGAL markedly rise within $3 \mathrm{hrs}$ of kidney injury compared to $\mathrm{SCr}$ which rises after $24 \mathrm{hrs}$. Among GpП subcategories, no significant difference was found in either pNGAL or kidney function parameters. Conclusions: pNGAL is an early biomarker of AKI and it can also discriminate type of AKI in cirrhosis
\end{abstract}

Keywords: Acute kidney injury, Cirrhosis, Acute tubular necrosis, Hepatorenal syndrome, Pre-renal azotemi.

\section{INTRODUCTION}

A cute kidney injury (AKI) is very common in patients with advanced cirrhosis; the most common causes are pre-renal azotemia (PRA) due to volume depletion, acute tubular necrosis (ATN) and hepatorenal syndrome (HRS), with prevalence rates of $68 \%, 33 \%$, and $25 \%$ respectively. [1] Because renal dysfunction is directly linked to the mortality rate of cirrhotic patients, a precise assessment of renal function is required to estimate the prognosis and determine the correct therapeutic intervention and response. ${ }^{[2]}$

Serum creatinine ( $\mathrm{SCr}$ ) could not represent GFR in several conditions, especially in liver disease ${ }^{[3]}$ as it is synthesized in the liver so any cause of hepatic parenchymal dysfunction will directly reduce creatine production. ${ }^{[4]}$ Therefore, $\mathrm{SCr}$ may overestimate renal function in these patients and normal SCr level cannot exclude early renal dysfunction in patients with liver cirrhosis. ${ }^{[5]}$

So we aimed in this work to study the usefulness of plasma Neutrophil gelatinase associated lipocalin (pNGAL) as an early biomarker of tubular damage and in the differential diagnosis of impairment of kidney function in cirrhosis.

\section{SUBJECTS AND METHODS}

This study has been conducted in collaboration between the Hepatology outpatient clinic, Al Ahrar hospital, Zagazig, Ministry of Health and the Internal Medicine outpatient clinic and the Hepatology Intensive Care Unit, Faculty of Medicine, Zagazig university hospitals during the period from April 2011 to April 2013.

It included 70 hepatic patients: 40 without $\mathrm{AKI}$ and 30 with AKI, in addition to 10 healthy control subjects. Their ages ranged from 38 to 54 yrs old with mean \pm SD $46.7 \pm 3.91$ years and 47 of them are males and other 33 are females.

Inclusion criteria:

Patients who were diagnosed to have chronic liver disease were included in this study either with AKI or not.

Exclusion criteria:

Patient with infection especially urinary tract infection, hypertension, diabetes, cardiac, hematologic, immunologic, malignant diseases were excluded from this study. 
Subjects were classified into 3 main groups:

- Group I (control GpI): It included 10 healthy volunteer control subjects ( 7 male and 3 female), their age ranged from (38 -50) year, with $\mathrm{X}$ (mean) $\pm \mathrm{SD}($ standard deviation $)=44.9 \pm 3.35$ year .

- Group П (GpП): compensated hepatic patients without AKI: It included 40 patients (22 male and 18 female), their age ranged from $(40-53)$ year, with $\mathrm{X} \pm \mathrm{SD}=46.4 \pm 3.82$ year. All of them had compensated liver disease \{clinically (no ascites or oedema), laboratory (normal serum albumin and no coagulopathy) radiologically (no ascites or portal hypertension) and endoscopically (no varices)

This group was further subdivided into four subcategories according to the cause of hepatic impairment:

(GpПa1): Hepatic patients with HCV under interferon plus ribavirin therapy. They were evaluated before starting the drug and 3 months after starting it. It included 10 patients ( 6 male and 4 female), their age ranged from $(42-52)$ year, with $\mathrm{X} \pm \mathrm{SD}=46.9 \pm 3.18$ year.

(GpПa2): Hepatic patients with HCV not under interferon or ribavirin therapy. It included 10 patients (5 male and 5 female), their age ranged from $(42-52)$ year, with $\mathrm{X} \pm \mathrm{SD}=47.3 \pm 3.68$ year.

(GpПb): Hepatic patients with Bilharzial liver fibrosis. It included 10 patients $(7$ male and 3 female), their age ranged from (40-52) year, with $\mathrm{X} \pm \mathrm{SD}=46.1 \pm 4.15$ year.

(GpПc): Hepatic patients with combined $\mathrm{HCV}$ and Bilharzial liver fibrosis. It included 10 patients (4 male and 6 female), their age ranged from $(40-53)$ year, with $\mathrm{X} \pm \mathrm{SD}=44.3 \pm 4.45$ year.

- Group II (GpWI): Decompensated hepatic patients with AKI: It included 30 patients (18 male and 12 female), their age ranged from (42-54) year, with $\mathrm{X} \pm \mathrm{SD}=47.7 \pm 4.01$ year. All of them had decompensated liver disease \{clinically (ascites and oedema), laboratory (low serum albumin and coagulopathy) radiologically (ascites and portal hypertension) and endoscopy (esophageal varices). This group was further subdivided into three subcategories according to the cause of kidney injury:

(Gp Шa): Acute tubular necrosis (ATN) OR intrinsic $A K I$ (iAKI). It included 10 patients (6 male and 4 female), their age ranged from (42-54) year, with $\mathrm{X} \pm \mathrm{SD}=49.3 \pm 4.14$ year.

(Gp Шb): Hepatorenal syndrome (HRS). It included 10 patients suspected at $1^{\text {st }}$ presentation and later on proved to have HRS. (7 male and 3 female), their age ranged from (42- 51) year, with $\mathrm{X} \pm \mathrm{SD}=47.7 \pm 3.34$ year.

(Gp Wc): Pre-renal azotemia (PRA) due to volume depletion. It included 10 patients $(5$ male and 5 female), their age ranged from (42- 52) year, with $\mathrm{X} \pm \mathrm{SD}=46.2 \pm 4.29$ year.

AKI is diagnosed according to the final consensus proposal of the Working Party that accept the definition of AKI in cirrhosis as an increase in serum creatinine of $>50 \%$ from baseline or a rise in $\mathrm{sCr}$ of $\geq 0.3 \mathrm{mg} / \mathrm{dl}$ in < $48 \mathrm{~h}$.Type $1 \mathrm{HRS}$ can be regarded as a specific form of AKI . Chronic kidney disease defined as GFR of $<60 \mathrm{ml} / \mathrm{min}$ for $>$ 3 months calculated using MDRD 6 formula. HRS ${ }_{[6]}^{\text {type }} 2$ is a specific form of chronic kidney disease.

All participants were subjected to the following:-

- Full history taking and detailed clinical examination with particular consideration on symptoms and sings of AKI.

- Routine laboratory investigations.

- Complete blood picture using sysmex S.F3000 automated analyzer.

- Liver function tests using Dimnsion E.S. chemical auto analyzer.

- Kidney function tests using Dimnsion E.S. chemical auto analyzer, itwas measured 2 times in (group Па1); before starting Interferon and Ribavirin therapy and 3 months after. Also, measured 3 times in ATN (group Шa); basal (before AKI), after 3 hrs and after 24 hrs of AKI.

- Estimation of GFR using Modification of Diet in Renal Disease (MDRD) equation.

- Viral hepatitis markers: hepatits B surface Antigen (HBsAg) and hepatitis $\mathrm{C}$ antibodies(HCV Abs). $\mathrm{HCV}$ polymerase chain reaction (HCV- PCR) Quantitative

- Complete Urine analysis and (Urine $\mathrm{Na}$ in patients with AKI)

- Liver biopsy when available.

- Abdominal ultrasonography .

- Specific laboratory investigations: Measurement of pNGAL by Enzyme linked immunosorbent assay (ELISA). Using human lipocalin-2/NGAL immunoassay, catalog number DLCN2, manufactured by R\&D Systems, Inc. 614 McKinley Place NE Minneapolis, MN 55413 United States of America. NGAL was measured 2 times in (group Пa1); before starting Interferon and Ribavirin therapy and 3 months after. Also, measured 3 times 
in ATN (group Шa); basal (before AKI), after 3 hrs and $24 \mathrm{hrs}$ of AKI.

\section{RESULTS \\ 1- Study of the demographic data among studied groups:}

Table (1) shows the demographic data among the studied groups. The hepatic patients without AKI group comprised 18 women and 22 men while hepatic patients with AKI group comprised 12 women and 18 men, the sex distribution didn't differ significantly between the two groups. The age rang in the group without AKI was (40-53) years while it was in the group with AKI (42-54), the mean age in the group without AKI was $(46.4 \pm 3.82)$ while it was $(47.7 \pm 4.01)$ in the group with AKI. The mean age didn't differ significantly between the two groups.

2- Study of the biochemical parameters among the groups:

Table (2) shows comparison between the groups of the study as regards their biochemical parameters using analysis of variance (ANOVA test). High significant difference was found in ALT, AST, serum albumin, PT, PTT, INR, WBCs, HB and platelets between studied groups $(\mathrm{P}<0.0001)$.

3- Study of the kidney functions parameters among the groups:

Table (3) shows there was high significant difference in pNGAL means among the studied groups, patients with AKI $(\boldsymbol{G p} \boldsymbol{W})$ had higher pNGAL compared to patients without AKI ( $G p I$ and $G p \boldsymbol{I}) \mathrm{P}<0.0001$. There was no significant difference in either $\mathrm{SCr}$ or blood urea or GFR means among the studied groups $(\mathrm{P}>0.05)$.

Table (4) shows no statistical significant difference among GpП subcategories as regarding pNGAL, sCr, blood urea and GFR. $(\mathrm{P}>0.05)$.

Table (5) shows comparison between $(\mathrm{X} \pm \mathrm{SD})$ of values among group Пa patients before taking interferon plus ribavirin therapy and 3 months after the therapy using paired $t$ test. High significant difference was found in AST, AST, WBCs, HB, platelets $(\mathrm{P}<0.0001)$. No significant difference was found in pNGAL, SCr, blood urea, GFR and S.albumin. $(\mathrm{P}>0.05)$.

Table (6) shows high significant difference in pNGAL means among subcategories of GpWI (P $<0.0001)$, patients with ATN (GpHa) had highest NGAL means $(295 \pm 19.08)$, patients with HRS $(\boldsymbol{G p} \boldsymbol{W} \boldsymbol{b})$ had intermediate NGAL means

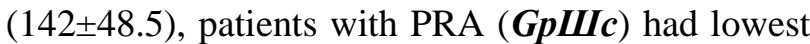
NGAL means $(86.5 \pm 11.22)$. On the other hand, there was no statistical significant difference among GpII subcategories as regarding SCr, blood urea and GFR. (P > 0.05).

Table (7) shows time-course of pNGAL and kidney function parameters in patients with ATN (GpШa). There was significant difference along the time course of NGAL (after 3 hours of AKI), sCr, blood urea and GFR (after 24 hours of AKI).

Table (8) shows comparison between the basal and 3 hours values of NGAL and kidney function parameters in patients with ATN (GpШa) using paired t test. There was high statistical significant increase in $\operatorname{pNGAL}(\mathrm{P}<0.0001)$, while there was no significant increase in $\mathrm{sCr}$, blood urea and GFR in those patients $(\mathrm{P}>0.05)$.

Table (9) shows comparison between the basal and 24 hours values of NGAL and kidney function parameters in patients with ATN (GpШa) using paired t test. There was high statistical significant increase in pNGAL, sCr, blood urea and GFR in those patients $(\mathrm{P}<0.0001)$

Table (10) shows validity of NGAL as a predictor of AKI within 3 hours. Setting a cutoff value of 134 $\mathrm{ng} / \mathrm{dl}$ for pNGAL yielded a sensitivity and specificity of $98.1 \%$ and $91.9 \%$ respectively with positive predictive value (PPV) of $55.4 \%$ and predictive value negative (NPV) of $96.7 \%$.

4-correlation and regression analysis:

Table (11) shows correlation between NGAL and other parameters among the studied groups. In both control subjects and hepatic patients with AKI there was no significant correlation between pNGAL levels and all other parameters $(\mathrm{p}<0.05)$. In hepatic patients with AKI, (After 3 hours of $\boldsymbol{A K I}$ ), there was no significant correlation between pNGAL levels and all other parameters except ALT and AST (positive correlation) and S.albumin (negative correlation). $(\mathrm{p}<0.05)$. Linear regression analysis shows that ALT and platelets were the most important independent factors related to pNGAL. (Table 12)

Table (13) shows that after $\mathbf{2 4}$ hours of AKI, there was significant positive correlation between pNGAL and $\mathrm{sCr}$.Also, significant negative correlation between pNGAL and GFR. $(\mathrm{p}<0.05)$. Linear regression analysis shows that $\mathrm{SCr}$ was the most important independent factor related to pNGAL. (Table 14) Fig.(1). 
Table (1) Demographic Data and classification of studied population using analysis of variance (ANOVA test) .

\begin{tabular}{|c|c|c|c|c|c|c|c|c|c|}
\hline \multirow[t]{2}{*}{ Group } & & \multirow[t]{2}{*}{$\mathbf{N}$} & \multicolumn{2}{|c|}{ Sex } & \multirow[t]{2}{*}{$\bar{F}$} & \multirow[t]{2}{*}{$\overline{\mathbf{P}}$} & \multirow{2}{*}{$\begin{array}{l}\text { Age range (year) } \\
\mathrm{X} \pm \mathrm{SD}\end{array}$} & \multirow[t]{2}{*}{$\mathbf{F}$} & \multirow[t]{2}{*}{$\overline{\mathbf{P}}$} \\
\hline & & & $\mathbf{M}$ & $\mathbf{F}$ & & & & & \\
\hline \multicolumn{2}{|l|}{ Group I } & 10 & 7 & 3 & \multirow[t]{3}{*}{0.376} & \multirow[t]{3}{*}{$\begin{array}{l}0.688 \\
\text { NS }\end{array}$} & $\begin{array}{l}38-50 \\
44.9 \pm 3.35\end{array}$ & \multirow[t]{3}{*}{2.300} & \multirow[t]{3}{*}{$\begin{array}{l}0.107 \\
\text { NS }\end{array}$} \\
\hline \multicolumn{2}{|l|}{ Group II } & 40 & 22 & 18 & & & $\begin{array}{l}40-53 \\
46.4 \pm 3.82\end{array}$ & & \\
\hline \multicolumn{2}{|l|}{ Group Ш } & 30 & 18 & 12 & & & $\begin{array}{l}42-54 \\
47.7 \pm 4.01\end{array}$ & & \\
\hline \multirow{4}{*}{  } & Па1 & 10 & 6 & 4 & \multirow[t]{4}{*}{1.180} & \multirow[t]{4}{*}{$\begin{array}{l}0.331 \\
\text { NS }\end{array}$} & $\begin{array}{l}42-52 \\
46.9 \pm 3.18\end{array}$ & \multirow[t]{4}{*}{0.519} & \multirow[t]{4}{*}{$\begin{array}{l}0.672 \\
\mathrm{NS}\end{array}$} \\
\hline & Па2 & 10 & 5 & 5 & & & $\begin{array}{l}42-52 \\
47.3 \pm 3.68\end{array}$ & & \\
\hline & $\Pi \mathbf{\Pi b}$ & 10 & 7 & 3 & & & $\begin{array}{l}40-52 \\
46.1 \pm 4.15\end{array}$ & & \\
\hline & Пс & 10 & 4 & 6 & & & $\begin{array}{l}40-53 \\
45.3 \pm 4.45\end{array}$ & & \\
\hline \multirow{3}{*}{ 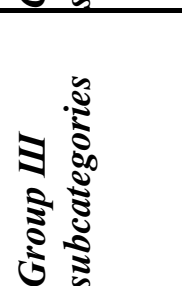 } & Ша & 10 & 6 & 4 & \multirow[t]{3}{*}{0.386} & \multirow[t]{3}{*}{$\begin{array}{l}0.684 \\
\text { NS }\end{array}$} & $\begin{array}{l}42-54 \\
49.3 \pm 4.14\end{array}$ & \multirow[t]{3}{*}{1.546} & \multirow[t]{3}{*}{$\begin{array}{l}0.231 \\
\mathrm{NS}\end{array}$} \\
\hline & UIb & 10 & 7 & 3 & & & $\begin{array}{l}42-51 \\
47.7 \pm 3.34\end{array}$ & & \\
\hline & Шॉc & 10 & 5 & 5 & & & $\begin{array}{l}42-52 \\
46.2 \pm 4.29\end{array}$ & & \\
\hline
\end{tabular}

Table (2): $(\mathrm{X} \pm \mathrm{SD})$ of values of studied Laboratory data among the different groups of patients using analysis of variance (ANOVA test).

\begin{tabular}{|c|c|c|c|c|c|}
\hline & $\begin{array}{l}\text { Group I } \\
X \pm S D \\
\text { Range }\end{array}$ & $\begin{array}{l}\text { Group I } \\
X \pm S D \\
\text { Range }\end{array}$ & $\begin{array}{l}\text { Group Ш } \\
X \pm S D \\
\text { Range }\end{array}$ & F-test & $P$-value \\
\hline $\begin{array}{l}A L T \\
(7-41 I U / L)\end{array}$ & $\begin{array}{l}16.0 \pm 7.82 \\
7-33\end{array}$ & $\begin{array}{l}73.1 \pm 10.4 \\
49-89\end{array}$ & $\begin{array}{l}79.1 \pm 14.7 \\
45-99\end{array}$ & 110.3 & $\begin{array}{l}<0.0001 \\
\text { HS }\end{array}$ \\
\hline $\begin{array}{l}A S T \\
(7-41 I U / L)\end{array}$ & $\begin{array}{l}17.1 \pm 5.99 \\
8-27 \\
\end{array}$ & $\begin{array}{l}63.5 \pm 12.2 \\
43-84\end{array}$ & $\begin{array}{l}96.7 \pm 19.3 \\
63-130 \\
\end{array}$ & 116.4 & $\begin{array}{l}<0.0001 \\
\mathrm{HS}\end{array}$ \\
\hline S. Albumin $(3.5-5 \mathrm{gm} / \mathrm{dl})$ & $\begin{array}{l}4.65 \pm 0.66 \\
3.6-5.9\end{array}$ & $\begin{array}{l}4.62 \pm 0.57 \\
3.5-5.9\end{array}$ & $\begin{array}{l}2.37 \pm 0.40 \\
1.6-3.1\end{array}$ & 174.6 & $\begin{array}{l}<0.0001 \\
\text { HS }\end{array}$ \\
\hline$P T(12.7-15.4$ sec $)$ & $\begin{array}{l}12.8 \pm 0.07 \\
12.7-12.9\end{array}$ & $\begin{array}{l}14.3 \pm 0.58 \\
12.8-15.4\end{array}$ & $\begin{array}{l}21.6 \pm 2.03 \\
16.9-25.3\end{array}$ & 321.6 & $\begin{array}{l}<0.0001 \\
\text { HS }\end{array}$ \\
\hline$P T T(26.3-39.4 \mathrm{sec})$ & $\begin{array}{l}30.4 \pm 2.46 \\
27-34 \\
\end{array}$ & $\begin{array}{l}37.3 \pm 1.81 \\
33-43 \\
\end{array}$ & $\begin{array}{l}42.2 \pm 2.98 \\
38-49 \\
\end{array}$ & 98.38 & $\begin{array}{l}<0.0001 \\
\text { HS }\end{array}$ \\
\hline $\operatorname{INR}(0.8-1.2)$ & $\begin{array}{l}1.0 \pm 0.005 \\
1-1.01\end{array}$ & $\begin{array}{l}1.11 \pm 0.05 \\
1.00-1.20\end{array}$ & $\begin{array}{l}1.69 \pm 0.18 \\
1.32-1.98\end{array}$ & 246.0 & $\begin{array}{l}<0.0001 \\
\text { HS }\end{array}$ \\
\hline$W B C(4.4-11.0 \times 10(9) / L)$ & $\begin{array}{l}7.77 \pm 1.69 \\
4.90-9.90\end{array}$ & $\begin{array}{l}6.45 \pm 1.27 \\
3.8-9.1\end{array}$ & $\begin{array}{l}5.48 \pm 1.49 \\
3.20-8.10\end{array}$ & 10.75 & $\begin{array}{l}<0.0001 \\
\text { HS }\end{array}$ \\
\hline $\begin{array}{l}H b \\
(12-17 \mathrm{~g} / \mathrm{dl})\end{array}$ & $\begin{array}{l}14.2 \pm 0.81 \\
12.9-15.3\end{array}$ & $\begin{array}{l}12.4 \pm 0.99 \\
10.9-14.4\end{array}$ & $\begin{array}{l}9.62 \pm 0.48 \\
8.9-10.7\end{array}$ & 157.5 & $\begin{array}{l}<0.0001 \\
\text { HS }\end{array}$ \\
\hline Platlets $(150-450 \times 10(9) / L)$ & $\begin{array}{l}250 \pm 72.6 \\
175-377\end{array}$ & $\begin{array}{l}172 \pm 11.7 \\
155-200\end{array}$ & $\begin{array}{l}92.3 \pm 20.7 \\
49-130\end{array}$ & 129.1 & $\begin{array}{l}<0.0001 \\
\mathrm{HS}\end{array}$ \\
\hline
\end{tabular}


Table (3): $(\mathrm{X} \pm \mathrm{SD})$ of values of kidney function parameters among the different groups using analysis of variance (ANOVA test).

\begin{tabular}{|c|c|c|c|c|c|}
\hline & $\begin{array}{l}\text { Group I } \\
X \pm S D \\
\text { Range }\end{array}$ & $\begin{array}{l}\text { Group } \Pi \\
X \pm S D \\
\text { Range }\end{array}$ & $\begin{array}{l}\text { Group Ш } \\
X \pm S D \\
\text { Range }\end{array}$ & $F$-test & $P$-value \\
\hline $\begin{array}{l}\text { NGAL } \\
(35-134 n g / d l)\end{array}$ & $\begin{array}{l}79.3 \pm 28.1 \\
45-130\end{array}$ & $\begin{array}{l}80.8 \pm 22.0 \\
43-125\end{array}$ & $\begin{array}{l}174.8 \pm 94.5 \\
69-318\end{array}$ & 22.55 & $\begin{array}{l}<0.0001 \\
\text { HS }\end{array}$ \\
\hline $\begin{array}{l}S C r \\
(0.5-1.4 \mathrm{mg} / \mathrm{dl})\end{array}$ & $\begin{array}{l}0.83 \pm 0.07 \\
0.7-0.9 \\
\end{array}$ & $\begin{array}{l}0.82 \pm 0.06 \\
0.7-0.9 \\
\end{array}$ & $\begin{array}{l}0.85 \pm 0.06 \\
0.75-0.96 \\
\end{array}$ & 2.25 & $\begin{array}{l}0.112 \\
\mathrm{NS} \\
\end{array}$ \\
\hline $\begin{array}{l}\text { Urea } \\
(15-50 \mathrm{mg} / \mathrm{dl})\end{array}$ & $\begin{array}{l}21.9 \pm 6.08 \\
15-31\end{array}$ & $\begin{array}{l}24.0 \pm 6.00 \\
12-34\end{array}$ & $\begin{array}{l}21.6 \pm 3.31 \\
15-27\end{array}$ & 2.08 & $\begin{array}{l}0.131 \\
\text { NS }\end{array}$ \\
\hline $\begin{array}{l}\text { GFR }(90-130 \mathrm{~mL} / \mathrm{min} / 1.73 \\
\left.\mathrm{m}^{2}\right)\end{array}$ & $\begin{array}{l}106 \pm 12.1 \\
90-130\end{array}$ & $\begin{array}{l}96.4 \pm 16.7 \\
69.6-126\end{array}$ & $\begin{array}{l}92.4 \pm 13.9 \\
67.6-118\end{array}$ & 2.87 & $\begin{array}{l}0.062 \\
\text { NS }\end{array}$ \\
\hline
\end{tabular}

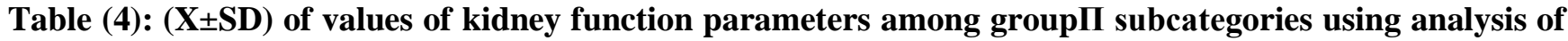
variance (ANOVA test).

\begin{tabular}{|c|c|c|c|c|c|c|}
\hline & $\begin{array}{l}\text { Group Пa1 } \\
X \pm S D \\
\text { Range } \\
\end{array}$ & $\begin{array}{l}\text { Group Пa2 } \\
X \pm S D \\
\text { Range } \\
\end{array}$ & $\begin{array}{l}\text { Group Пb } \\
X \pm S D \\
\text { Range } \\
\end{array}$ & $\begin{array}{l}\text { Group Пc } \\
X \pm S D \\
\text { Range } \\
\end{array}$ & $F$-test & $\begin{array}{l}P \\
\text { value }\end{array}$ \\
\hline $\begin{array}{l}\text { NGAL } \\
(35-134 n g / d l)\end{array}$ & $\begin{array}{l}76.6 \pm 11.0 \\
60-95\end{array}$ & $\begin{array}{l}79.6 \pm 24.2 \\
44-120\end{array}$ & $\begin{array}{l}85.1 \pm 24.0 \\
48-120\end{array}$ & $\begin{array}{l}81.8 \pm 27.8 \\
43-125\end{array}$ & 0.177 & $\begin{array}{l}0.949 \\
\text { NS }\end{array}$ \\
\hline $\begin{array}{l}S C r \\
(0.5-1.4 \mathrm{mg} / \mathrm{dl})\end{array}$ & $\begin{array}{l}0.80 \pm 0.03 \\
0.7-0.8\end{array}$ & $\begin{array}{l}0.83 \pm 0.07 \\
0.7-0.9\end{array}$ & $\begin{array}{l}0.81 \pm 0.06 \\
0.7-0.9\end{array}$ & $\begin{array}{l}0.83 \pm 0.07 \\
0.7-0.9\end{array}$ & 0.691 & $\begin{array}{l}0.602 \\
\text { NS }\end{array}$ \\
\hline $\begin{array}{l}\text { Urea } \\
(15-50 \mathrm{mg} / \mathrm{dl})\end{array}$ & $\begin{array}{l}23.4 \pm 5.56 \\
15-31\end{array}$ & $\begin{array}{l}26.2 \pm 6.32 \\
17-34\end{array}$ & $\begin{array}{l}24.4 \pm 5.19 \\
17-32\end{array}$ & $\begin{array}{l}22 \pm 6.90 \\
12-33\end{array}$ & 0.883 & $\begin{array}{l}0.482 \\
\mathrm{NS}\end{array}$ \\
\hline $\begin{array}{l}\text { GFR }(90-130 \\
\left.\mathrm{mL} / \mathrm{min} / 1.73 \mathrm{~m}^{2}\right)\end{array}$ & $\begin{array}{l}99.4 \pm 16.4 \\
78.4-123\end{array}$ & $\begin{array}{l}92.3 \pm 15.8 \\
69.8-112\end{array}$ & $\begin{array}{l}103.1 \pm 16.7 \\
70.4-126\end{array}$ & $\begin{array}{l}90.6 \pm 17.2 \\
69.9-126\end{array}$ & 1.747 & $\begin{array}{l}0.156 \\
\text { NS }\end{array}$ \\
\hline
\end{tabular}


Table (5):Comparison between $(\mathrm{X} \pm \mathrm{SD})$ of values among group Пa patients before taking interferon plus ribavirin therapy and 3 months after the therapy using paired t test.

\begin{tabular}{|c|c|c|c|c|}
\hline & Before & After & $t$ & $\mathbf{P}$ \\
\hline $\begin{array}{l}\text { NGAL } \\
(35-134 \mathrm{ng} / d l)\end{array}$ & $\begin{array}{l}76.6 \pm 10.9 \\
60-90\end{array}$ & $\begin{array}{l}80.4 \pm 23.4 \\
44-130\end{array}$ & -0.644 & $\begin{array}{l}0.536 \\
\mathrm{NS}\end{array}$ \\
\hline $\begin{array}{l}\text { Creatinine } \\
(0.5-1.4 \mathrm{mg} / \mathrm{dl})\end{array}$ & $\begin{array}{l}0.79 \pm 0.02 \\
0.73-0.82 \\
\end{array}$ & $\begin{array}{l}0.80+0.02 \\
0.73-0.83 \\
\end{array}$ & -0.921 & $\begin{array}{l}0.381 \\
\mathrm{NS}\end{array}$ \\
\hline $\begin{array}{l}\text { Urea } \\
(15-50 \mathrm{mg} / \mathrm{dl})\end{array}$ & $\begin{array}{l}23.4+5.5 \\
15-31 \\
\end{array}$ & $\begin{array}{l}27.3+6.53 \\
20-40\end{array}$ & -1.78 & $\begin{array}{l}0.108 \\
\mathrm{NS}\end{array}$ \\
\hline $\begin{array}{l}\text { GFR }(90-130 \mathrm{~mL} / \mathrm{min} / 1.73 \\
\left.\mathrm{m}^{2}\right)\end{array}$ & $\begin{array}{l}99.3+16.4 \\
78-123\end{array}$ & $\begin{array}{l}99+16.5 \\
76.7-123.4\end{array}$ & 0.544 & $\begin{array}{l}0.600 \\
\mathrm{NS}\end{array}$ \\
\hline $\begin{array}{l}A L T \\
(7-41 I U / L) \\
\end{array}$ & $\begin{array}{l}85.5+3.13 \\
80-89 \\
\end{array}$ & $\begin{array}{l}37.9+2.92 \\
33-42 \\
\end{array}$ & -33.4 & $\begin{array}{l}<0.0001 \\
\mathrm{HS}\end{array}$ \\
\hline $\begin{array}{l}A S T \\
(7-41 I U / L)\end{array}$ & $\begin{array}{l}79.7+2.05 \\
77-84 \\
\end{array}$ & $\begin{array}{l}37.7+1.70 \\
35-40\end{array}$ & -50.6 & $\begin{array}{l}<0.0001 \\
\mathrm{HS}\end{array}$ \\
\hline $\begin{array}{l}\text { Albumin } \\
(3.5-5 \mathrm{gm} / \mathrm{dl})\end{array}$ & $\begin{array}{l}4.47+0.54 \\
3.5-5.3 \\
\end{array}$ & $\begin{array}{l}4.41+0.30 \\
3.8-4.8 \\
\end{array}$ & 0.415 & $\begin{array}{l}0.688 \\
\mathrm{NS} \\
\end{array}$ \\
\hline $\begin{array}{l}W B C \\
(4.4-11.0 \times 10(9) / L)\end{array}$ & $\begin{array}{l}6.02+1.29 \\
4.0-7.8 \\
\end{array}$ & $\begin{array}{l}3.61+0.68 \\
2.4-4.5 \\
\end{array}$ & 7.38 & $\begin{array}{l}<0.0001 \\
\mathrm{HS}\end{array}$ \\
\hline $\begin{array}{l}H B \\
(12-17 \mathrm{~g} / \mathrm{dl})\end{array}$ & $\begin{array}{l}13.5+0.59 \\
12.4-14.4 \\
\end{array}$ & $\begin{array}{l}10.9+0.94 \\
9.3-12.1 \\
\end{array}$ & 10.89 & $\begin{array}{l}<0.0001 \\
\mathrm{HS}\end{array}$ \\
\hline $\begin{array}{l}\text { Platlets } \\
(150-450 \times 10(9) / L)\end{array}$ & $\begin{array}{l}188+8.45 \\
170-200 \\
\end{array}$ & $\begin{array}{l}109+16.4 \\
90-134 \\
\end{array}$ & 12.07 & $\begin{array}{l}<0.0001 \\
\mathrm{HS}\end{array}$ \\
\hline
\end{tabular}

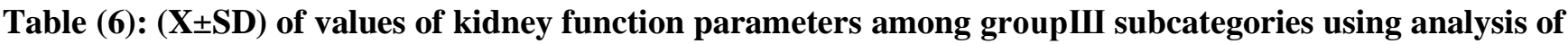
variance (ANOVA test).

\begin{tabular}{|c|c|c|c|c|c|}
\hline & $\begin{array}{l}\text { Group Ша } \\
X \pm S D \\
\text { Range }\end{array}$ & $\begin{array}{l}\text { Group Шb } \\
X \pm S D \\
\text { Range }\end{array}$ & $\begin{array}{l}\text { Group Шc } \\
X \pm S D \\
\text { Range }\end{array}$ & $F$-test & $P$-value \\
\hline $\begin{array}{l}\text { NGAL } \\
(35-134 n g / d l)\end{array}$ & $\begin{array}{l}295.3 \pm 19.08 \\
260-318\end{array}$ & $\begin{array}{l}142.7 \pm 48.5 \\
89-263\end{array}$ & $\begin{array}{l}86.5 \pm 11.2 \\
69-105\end{array}$ & 110.9 & $\begin{array}{l}<0.0001 \\
\text { HS }\end{array}$ \\
\hline $\begin{array}{l}S C r \\
(0.5-1.4 \mathrm{mg} / \mathrm{dl})\end{array}$ & $\begin{array}{l}0.85 \pm 0.07 \\
0.76-0.95\end{array}$ & $\begin{array}{l}0.87 \pm 0.06 \\
0.78-0.96\end{array}$ & $\begin{array}{l}0.82 \pm 0.05 \\
0.75-0.88\end{array}$ & 1.314 & $\begin{array}{l}0.285 \\
\mathrm{NS}\end{array}$ \\
\hline $\begin{array}{l}\text { Urea } \\
(15-50 \mathrm{mg} / \mathrm{dl})\end{array}$ & $\begin{array}{l}19.9 \pm 2.84 \\
15-25\end{array}$ & $\begin{array}{l}22.1 \pm 3.31 \\
18-26\end{array}$ & $\begin{array}{l}22.7 \pm 3.37 \\
19-27\end{array}$ & 0.876 & $\begin{array}{l}0.463 \\
\mathrm{NS}\end{array}$ \\
\hline $\begin{array}{l}\text { GFR (90-130 } \mathrm{mL} / \mathrm{min} / 1.73 \\
\left.\mathrm{~m}^{2}\right)\end{array}$ & $\begin{array}{l}91.5 \pm 12.9 \\
67.6-110\end{array}$ & $\begin{array}{l}91.9 \pm 13.4 \\
71-114\end{array}$ & $\begin{array}{l}93.8 \pm 16.5 \\
71.7-118\end{array}$ & 2.360 & $\begin{array}{l}0.088 \\
\mathrm{NS}\end{array}$ \\
\hline
\end{tabular}


Table (7):Time-course of NGAL and kidney function parameters in patients with ATN (GpШa).

\begin{tabular}{|c|c|c|c|c|c|}
\hline Biomarker & Basal & 3hs & $24 \mathrm{hs}$ & $\mathbf{f}$ & $\mathbf{p}$ \\
\hline $\begin{array}{l}\text { NGAL } \\
(35-134 n g / d l)\end{array}$ & $\begin{array}{l}76.6 \pm 20.9 \\
45-120\end{array}$ & $\begin{array}{l}295 \pm 19.08 \\
260-318\end{array}$ & $\begin{array}{l}187 \pm 31.89 \\
160-225\end{array}$ & 166 & $\begin{array}{l}<0.0001 \\
\mathrm{HS}\end{array}$ \\
\hline $\begin{array}{l}\text { Creatinine } \\
(0.5-1.4 \mathrm{mg} / \mathrm{dl})\end{array}$ & $\begin{array}{l}0.84 \pm 07 \\
0.75-0.95\end{array}$ & $\begin{array}{l}0.84 \pm 0.07 \\
0.76-0.95\end{array}$ & $\begin{array}{l}1.37 \pm 07 \\
1.25-150\end{array}$ & 139 & $\begin{array}{l}<0.0001 \\
\mathrm{HS}\end{array}$ \\
\hline $\begin{array}{l}\text { Urea } \\
(15-50 \mathrm{mg} / \mathrm{dl})\end{array}$ & $\begin{array}{l}18.5 \pm 2.32 \\
15-22\end{array}$ & $\begin{array}{l}19.9 \pm 2.85 \\
15-25\end{array}$ & $\begin{array}{l}36.7 \pm 5.56 \\
28-46\end{array}$ & 34.8 & $\begin{array}{l}<0.0001 \\
\mathrm{HS}\end{array}$ \\
\hline $\operatorname{GFR}\left(90-130 \mathrm{~mL} / \mathrm{min} / 1.73 \mathrm{~m}^{2}\right)$ & $\begin{array}{l}91.95 \pm 13.2 \\
67.6-110\end{array}$ & $\begin{array}{l}91.5 \pm 12.9 \\
67.6-110\end{array}$ & $\begin{array}{l}52.4 \pm 7.26 \\
39.9-60.7\end{array}$ & 38.9 & $\begin{array}{l}<0.0001 \\
\text { HS }\end{array}$ \\
\hline
\end{tabular}

Basal (before kidney injury) 3hs (after 3 hours of AKI)24hs(after 24 hours of AKI).

Table (8): Comparison between the basal and 3 hours values of NGAL and kidney function parameters in patients with ATN(GpWa) using paired t test.

\begin{tabular}{|c|c|c|c|c|}
\hline & Basal & $3 \mathrm{hrs}$ & $\mathbf{t}$ & $\bar{p}$ \\
\hline $\begin{array}{l}\text { NGAL } \\
(35-134 n g / d l)\end{array}$ & $\begin{array}{l}76.6 \pm 20.9 \\
45-120 \\
\end{array}$ & $\begin{array}{l}295 \pm 19.08 \\
260-318 \\
\end{array}$ & -18.943 & $\begin{array}{l}<0.0001 \\
\text { HS }\end{array}$ \\
\hline $\begin{array}{l}\text { Creatinine } \\
(0.5-1.4 \mathrm{mg} / \mathrm{dl})\end{array}$ & $\begin{array}{l}0.84 \pm 07 \\
0.75-0.95\end{array}$ & $\begin{array}{l}0.84 \pm 0.07 \\
0.76-0.95\end{array}$ & 1.912 & $\begin{array}{l}0.081 \\
\text { NS }\end{array}$ \\
\hline $\begin{array}{l}\text { Urea } \\
(15-50 \mathrm{mg} / \mathrm{dl})\end{array}$ & $\begin{array}{l}18.5 \pm 2.32 \\
15-22\end{array}$ & $\begin{array}{l}19.9 \pm 2.85 \\
15-25\end{array}$ & -1.769 & $\begin{array}{l}0.111 \\
\mathrm{NS}\end{array}$ \\
\hline GFR $\left(90-130 \mathrm{~mL} / \mathrm{min} / 1.73 \mathrm{~m}^{2}\right)$ & $\begin{array}{l}91.95 \pm 13.2 \\
67.6-110\end{array}$ & $\begin{array}{l}91.5 \pm 12.9 \\
67.6-110\end{array}$ & 1.964 & $\begin{array}{l}0.088 \\
\text { NS }\end{array}$ \\
\hline
\end{tabular}

Table (9): Comparison between the basal and 24 hours values of NGAL and kidney function parameters in patients with ATN (GpШa) using paired t test.

\begin{tabular}{|c|c|c|c|c|}
\hline & Basal & $24 \mathrm{hrs}$ & $t$ & p \\
\hline $\begin{array}{l}\text { NGAL } \\
(35-134 n g / d l)\end{array}$ & $\begin{array}{l}76.6 \pm 20.9 \\
45-120\end{array}$ & $\begin{array}{l}187 \pm 31.89 \\
160-225\end{array}$ & -9.278 & $\begin{array}{l}<0.0001 \\
\text { HS }\end{array}$ \\
\hline $\begin{array}{l}\text { Creatinine } \\
(0.5-1.4 \mathrm{mg} / \mathrm{dl})\end{array}$ & $\begin{array}{l}0.84 \pm 07 \\
0.75-0.95\end{array}$ & $\begin{array}{l}1.37 \pm 07 \\
1.25-150\end{array}$ & -31.741 & $\begin{array}{l}<0.0001 \\
\text { HS }\end{array}$ \\
\hline $\begin{array}{l}\text { Urea } \\
(15-50 \mathrm{mg} / \mathrm{dl})\end{array}$ & $\begin{array}{l}18.5 \pm 2.32 \\
15-22\end{array}$ & $\begin{array}{l}36.7 \pm 5.56 \\
28-46\end{array}$ & -10.181 & $\begin{array}{l}<0.0001 \\
\text { HS }\end{array}$ \\
\hline $\begin{array}{l}\text { GFR }(90-130 \mathrm{~mL} / \mathrm{min} / 1.73 \\
\left.\mathrm{m}^{2}\right)\end{array}$ & $\begin{array}{l}91.95 \pm 13.2 \\
67.6-110\end{array}$ & $\begin{array}{l}52.4 \pm 7.26 \\
39.9-60.7\end{array}$ & 16.106 & $\begin{array}{l}<0.0001 \\
\text { HS }\end{array}$ \\
\hline
\end{tabular}

Table (10): Validity of NGAL as a predictor of AKI within 3 hours

\begin{tabular}{lllll}
\hline Biomarker & Sensitivity & Specificity & PPV & NPV \\
\hline NGAL & 98.1 & 91.9 & 84.2 & 96.7 \\
\hline
\end{tabular}

Cutoff value of $\mathrm{NGAL}=134 \mathrm{ng} / \mathrm{dl}$. 
Table (11): Correlation coefficient (r) between NGAL and other parameters among the studied groups after 3 hours of kidney injury.

\begin{tabular}{|c|c|c|c|c|c|c|}
\hline & \multicolumn{2}{|l|}{$G p I$} & \multicolumn{2}{|l|}{ Gp II } & \multicolumn{2}{|l|}{ Gp $\amalg$} \\
\hline & $\mathrm{r}$ & $\mathrm{p}$ & $\mathrm{r}$ & $\mathrm{p}$ & $\mathrm{r}$ & $\mathrm{p}$ \\
\hline Creatinine & -0.304 & 0.393 & 0.226 & 0.161 & 0.241 & 200 \\
\hline Urea & -0.272 & 0.446 & -0.020 & 0.904 & -0.251 & 180 \\
\hline GFR & 0.360 & 0.308 & -0.092 & 0.571 & -0.070 & 712 \\
\hline$\overline{A L T}$ & -0.051 & 0.890 & -0.125 & 0.443 & 0.679 & $<0.0001(\mathrm{~S})$ \\
\hline$A S T$ & 0.298 & 0.402 & -0.051 & 0.756 & 0.689 & $<0.0001(\mathrm{~S})$ \\
\hline S. Albumin & -0.416 & 0.231 & 0.142 & 0.381 & -0.313 & 0.046 \\
\hline$P T$ & 0.063 & 0.863 & 0.177 & 0.274 & -0.223 & 235 \\
\hline$P T T$ & -0.560 & 0.092 & 0.242 & 0.132 & -0.220 & 0.242 \\
\hline INR & 0.271 & 0.449 & 0.178 & 0.271 & -0.114 & 0.574 \\
\hline$W B C$ & -0.018 & 0.960 & -0.175 & 0.280 & -0.098 & 0.607 \\
\hline HB & 0.345 & 0.329 & -0.173 & 0.287 & 0.291 & 0.119 \\
\hline Platelets & -0.568 & 0.086 & -0.122 & 0.452 & 0.137 & 0.460 \\
\hline
\end{tabular}

Table (12): Linear regression analysis between pNGAL and other parameters after 3 hours of kidney injury in hepatic patients with AKI (Gp III).

\begin{tabular}{lllll}
\hline \multicolumn{1}{l}{ Beta \pm Std. Error $(\boldsymbol{\beta} \pm \mathbf{S E})$} & $\mathbf{t}$ & $\mathbf{p}$ & \\
\hline GFR & $0.375 \pm 1.59$ & 0.235 & 0.820 & $\mathrm{NS}$ \\
\hline Urea & $-5.49 \pm 5.03$ & -1.09 & 0.307 & $\mathrm{NS}$ \\
\hline SCr & $-444 \pm 287.9$ & -1.54 & 0.162 & $\mathrm{NS}$ \\
\hline ALT & $7.70 \pm 1.82$ & 4.22 & 0.003 & $\mathrm{~S}$ \\
\hline AST & $-1.56 \pm 1.42$ & -1.101 & 0.303 & $\mathrm{NS}$ \\
\hline Albumin & $-43.16 \pm 48.98$ & -0.881 & 0.404 & $\mathrm{NS}$ \\
\hline WBCs & $-0.600 \pm 11.65$ & -0.051 & 0.960 & $\mathrm{NS}$ \\
\hline HB & $7.38 \pm 49.45$ & 0.149 & 0.885 & $\mathrm{NS}$ \\
\hline Platelets & $2.45 \pm 0.811$ & 3.02 & 0.017 & $\mathrm{~S}$ \\
\hline PT & $-30.74 \pm 24.05$ & -1.28 & 0.237 & $\mathrm{NS}$ \\
\hline PTT & $6.34 \pm 7.02$ & 0.903 & 0.393 & $\mathrm{NS}$ \\
\hline INR & $302.9 \pm 334.8$ & 0.905 & 0.392 & $\mathrm{NS}$ \\
\hline
\end{tabular}

Table (13): Correlation coefficient (r) between NGAL and kidney function parameters after 24 hours of kidney injury in hepatic patients with AKI (Gp $U$ )..

\begin{tabular}{lll}
\hline & \multicolumn{2}{l}{ Gp W (Decompensated hepatic patients with AKI) } \\
\cline { 2 - 3 } & $\mathrm{r}$ & $\mathrm{p}$ \\
\hline $\boldsymbol{S C r}$ & 0.810 & $<0.0001(\mathrm{~S})$ \\
\hline Urea & -0.075 & $0.695 \mathrm{NS}$ \\
\hline GFR & -0.512 & $0.004(\mathrm{~S})$ \\
\hline
\end{tabular}


Table (14): Linear regression analysis between pNGAL and kidney function parameters after 24 hours of kidney injury in hepatic patients with AKI (Gp $U$ ).

\begin{tabular}{lllll}
\hline & Beta \pm Std. Error $(\boldsymbol{\beta} \pm \mathbf{S E})$ & $\mathbf{t}$ & $\mathbf{p}$ & \\
\hline SCr & $438.720 \pm 72.306$ & 6.068 & $<0.0001$ & $\mathrm{~S}$ \\
\hline Urea & $-1.441 \pm .764$ & -1.886 & 0.070 & $\mathrm{NS}$ \\
\hline GFR & $.494 \pm .544$ & 0.907 & 0.373 & $\mathrm{NS}$ \\
\hline
\end{tabular}

Fig. (1): Curve estimation of relationship between SCr and NGAL in group III after 24 hours of AKI by linear regression analysis.

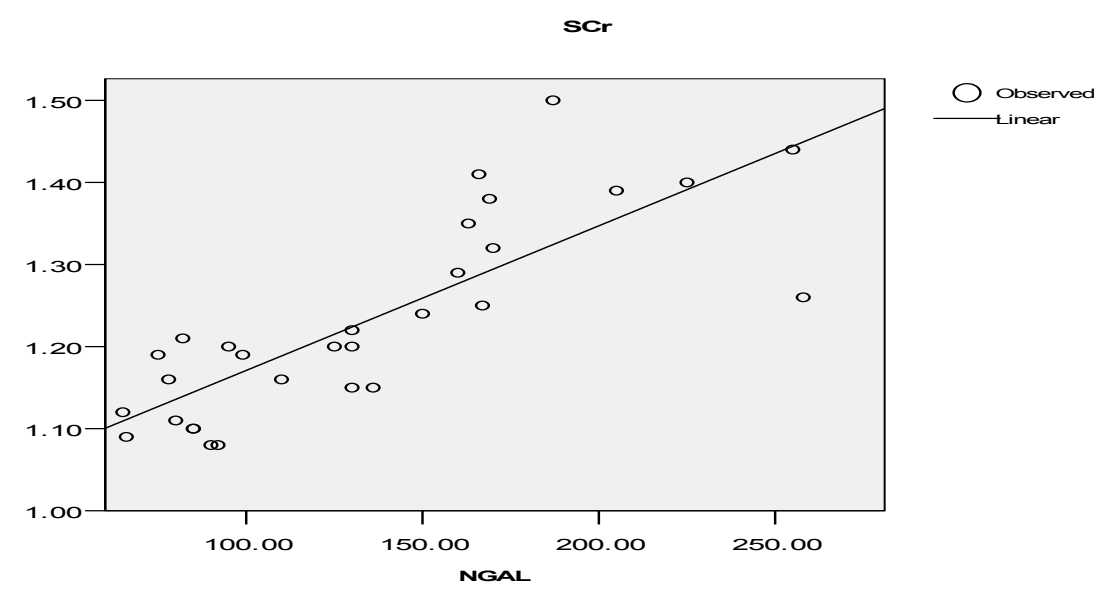

\section{DISCUSSION}

AKI in patients with cirrhosis is common and deadly. Up to $20 \%$ of hospitalized patients with cirrhosis develop AKI ${ }^{[1]}$ and once AKI occurs there is a reported fourfold increased risk of mortality ${ }^{[7]}$. In cirrhosis, AKI types include PRA, HRS and intrinsic acute kidney injury (iAKI) or ATN but their effect on mortality risk varies. ${ }^{[1]}$ The differential diagnosis of AKI types in cirrhosis is important to apply specific therapies for each cause. PRA should be treated with plasma volume expansion, while this is not effective and may be even deleterious in patients with ATN. ${ }^{[8]}$ ATN should in general be treated with Renal Replacement Therapy (RRT) while HRS should be treated with vasoconstrictors an albumin. ${ }^{[9]}$

Unfortunately these forms of AKI are difficult to distinguish clinically as $\mathrm{SCr}$, the clinical standard to define kidney function, poorly discriminates AKI type in cirrhosis [10], as it is associated with many problems: (A) $\mathrm{SCr}$ is influenced by age, gender and ethnicity. ${ }^{[11]}$ (B) SCr is a byproduct of the metabolism of creatine, which is stored in muscles, so it reflects endogenous muscle mass as well as protein intake. ${ }^{[4]}$ (C) It is synthesized in the liver, so any hepatic parenchymal dysfunction will directly reduce creatine production. [4] (D) The oedematous state in ascitic patients leading to creatinine dilution in the body with low serum levels. ${ }^{[12]}$ (E) SCr concentrations may not change until a significant amount of kidney function has already been lost, meaning that renal injury is already present or occurs before $\mathrm{SCr}$ is elevated. Also, the capacity of the kidneys to excrete creatinine is hardly predictable for the individual; it also depends on some medication interfering with tubular creatinine transport. Finally, during acute changes in glomerular filtration, $\mathrm{SCr}$ does not accurately depict kidney function until steady-state equilibrium has been reached, which may require several days. ${ }^{[13]}$

These factors render SCr less sensitive in reflecting renal dysfunction in cirrhotic patients; the sensitivity of SCr for reduced GFR may be as low as $18.5 \% .{ }^{[14]}$ Therefore, there is an urgent need for objective methods in the differential diagnosis of impairment of kidney function in cirrhosis.

In the last years, there has been a major interest in the field of nephrology to investigate substances either in the urine or blood, known as 
kidney biomarkers, that are released at the time of injury of tubular cells and could be used for an early diagnosis of acute kidney injury and also for differential diagnosis of impaired kidney function [15]. Among the different biomarkers, NGAL has received a great deal of attention. A number of studies have shown that NGAL predicts the development of kidney injury in different clinical settings, and may also be helpful in the prediction of the need for dialysis and early mortality. ${ }^{[16]}$ However, the existing information on the potential usefulness of NGAL in the differential diagnosis of impaired kidney function in cirrhosis is very scant. ${ }^{[17]}$ So, in our study, the usefulness of plasma NGAL ( pNGAL) as an early biomarker of AKI and as a biomarker for the differential diagnosis of causes of AKI in cirrhosis has been investigated.

Our study revealed that hepatic patients with AKI $(\boldsymbol{G p} \boldsymbol{W})$ had no significant different in kidney function parameters compared to hepatic patients without AKI and control. $(\mathrm{P}>0.05)$. This came in consistence with Sherman, D.S., et al. who proved that $\mathrm{SCr}$ in patients with decompensated cirrhosis can still be within the normal range despite significant renal dysfunction. ${ }^{[5]}$ Also, Nguyen, M.T and Devarajan,P. proved that $\mathrm{SCr}$ does not accurately depict kidney function until a steadystate equilibrium has been reached, which may require several days. ${ }^{[13]}$

On the other hand, pNGAL was significantly higher in hepatic patients with AKI compared to hepatic patients without AKI and control. $\mathrm{P}<0.0001$. The present study correlates with Portal, A.J., et al. who proved that pNGAL was significantly elevated in hepatic patients with AKI post liver transplantation (i.e decompasated cirrhosis) compared to those without AKI ( $\mathrm{P}<$ 0.001). ${ }^{[18]}$ Also, Fagundes, C., et al. proved that patients with cirrhosis and kidney dysfunction irrespective of aetiology had greater serum NGAL levels compared to those without kidney dysfunction. ${ }^{[19]}$

Among the 4 subcategories of group (II), ALT and AST were significantly higher than control group reflecting hepatocellular injury rather than synthetic liver function which is better reflected by albumin and prothrombin time ${ }^{[20]}$. (Which are not significantly different from control). Also, values of PT, PTT, INR, WBCs, $\mathrm{Hb}$ and platelets were significantly higher than in control group (yet all were within the normal range).such findings confirm the state of compensation in such patients .
Kidney function parameters (SCr, Urea and GFR) and pNGAL in such 4 subcategories were found not significantly different from control group or between the subcategories and some of them. i.e no renal deterioration was found. Our finding reflected and confirmed normal kidney function (no kidney injury) among them. Our finding came in consistence with the study of Gungor, G., et al. proved there was no significant difference between stable cirrhosis patients and control subjects in either kidney function parameters ( $\mathrm{SCr}$ and GFR) or pNGAL levels. $(p>05) .{ }^{[21]}$

Considering the subcategory IIal, our finding came in consistence with the study of Montalbano, M., et al. who proved that treatment with pegylated-IFN-alpha2b and ribavirin in a liver/kidney transplant recipient didn't affect kidney function. ${ }^{[22]}$

In the same subcategory IIa1, high significant difference was found in ALT, AST, WBCs, HB and platelets ( $\mathrm{P}<0.0001)$. table (5). This came in consistence with Wittho, Th, et al. who reported that median ALT and AST levels, (which were elevated as expected in patients suffering from hepatitis C), decreased during treatment and were within the normal range or slightly above at the end of treatment. Also, reported that the median values of WBCs and platelets as well as median haemoglobin concentration decreased during treatment with interferon therapy. ${ }^{[23]}$

Considering the subcategory IIa2, our finding came in consistence with the study of Kamal, S., et al. reported that patients with $\mathrm{HCV}$ alone (without concomitant bilharziasis) has less impairment in kidney function. ${ }^{[24]}$ Considering the subcategory $I I b$, our finding came in consistence with the study of Mahmoud, K.M., et al. that proved no significant difference in the incidence of acute and chronic rejection between schistosoma infected cases and control group. ${ }^{[25]}$ Considering the subcategory IIc, our study correlated with Abbas, O.M., et al. who reported that schistosoma coinfection could have a protective effect against mixed cryoglobulinaemia (CG) [in which renal disease more common than in Type $\mathrm{I}(\mathrm{CG})]$ in hepatitis $\mathrm{C}$ patients, so less renal impairment. ${ }^{[26]}$ However, in contrary to our study, Kamal, S., et al. reported that patients with concomitant $\mathrm{HCV}$ and bilharziasis had a marked increase in the ChildPugh score (more advanced liver disease), more frequent episodes of upper gastrointestinal bleeding, more renal impairment. ${ }^{[2]}$ All these factors not 
present in our patients (our patients in group II were compensated, no gastrointestinal bleeding, so no renal abnormality found.

Among the subcategories of group (III), ALT and AST were significantly higher than control group (reflecting hepatocellular injury) associated with evidence of impaired hepatic synthetic function (low serum albumin, elevated PT, $P T T$, INR significantly different from compensated group II and control subjects) and portal hypertension (splenomegaly with low WBCs, $\mathrm{Hb}$, platelets significantly different from compensated group II and control subjects) ${ }^{[20]}$.All were present in such patients of group III confirming the state of decompensation in such patients.

Kidney function parameters in such 3 subcategories were found not significantly different between the subcategories and some of them $(\mathrm{P}>$ 0.05 ), our finding correlated with the study of Garcia-Tsao, G., et al. ${ }^{[1]}$ that reported $\mathrm{SCr}$ is not helpful in distinguishing various causes of renal injury. Also, Verna,E.C., et al. ${ }^{[27]}$ proved that $\mathrm{SCr}$ in patients with iAKI was statistically similar to those with PRA and HRS.

pNGAL was significantly different between the subcategories and some of them $(\mathrm{P}<0.0001)$, patients with ATN (GpHa) had highest NGAL

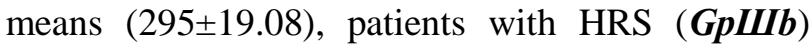
had intermediate NGAL means $(142 \pm 48.5)$, patients with PRA (GpHc) had lowest NGAL means $(86.5 \pm 11.22)$.

The mechanism by which patients with HRS have intermediate pNGAL levels remains uncertain. HRS physiology is classically thought to be an extreme prerenal state ${ }^{[28]}$ with severe renovascular vasoconstriction and decreased GFR, but normal intrinsic kidney function. Kidney function can return to normal after improvement of hepatic hemodynamics (i.e. TIPS, surgical portalcaval shunts and liver transplant) ${ }^{[29]}$ or after transplantation of the kidney into a recipient with normal hepatic function ${ }^{[30]}$. However, pathologic investigations have reported subtle kidney tubular and glomerular damage in HRS kidneys, some seen only with electron microscopy ${ }^{[31]}$, perhaps resulting from the cellular changes associated with chronic activation of angiotensin-aldosterone signaling ${ }^{[32]}$.So, HRS could not be enterely functional in nature but may be associated to tubular damage.

Our study correlates with Niculae, A. et al. ${ }^{[33]}$ who observed that NGAL serum values increased significantly in patients with different etiologies of acute renal failure, as compared to those diagnosticated later with hepatorenal syndrome (HRS). Similar results was found in other studies used urinary NGAL (uNGAL) as diagnostic tool in the differential diagnosis of impaired kidney function in hepatic patients of them the studies of Verna, E.C., ${ }^{[27]}$ et al. Fagundes, C., et al. ${ }^{[19]}$ and El-Bassat, H.,et al. ${ }^{[34]}$ all proved that patients with ATN had the highest values of UNGAL, while patients with pre-renal azotemia had the lowest values, patients with HRS had intermediate values.

In patients with ATN (GpHa), there was significant difference along the time course of pNGAL and kidney function parameters, however, pNGAL shows high significant rise within $3 \mathrm{hrs}$ of kidney injury compared to kidney function parameters that rise after 24 hours. These values came in consistence with the findings of Mori $\mathbf{K}$ and Nakao $\mathbf{K}$ who observed early rise of NGAL in the course of AKI, prior to $\mathrm{SCr}$ elevation ${ }^{[35]}$.

\section{CONCLUSION}

* pNGAL is an early biomarker of AKI and it can also discriminate type of AKI in cirrhosis.

* HRS could not be enterely functional in nature but may be associated to tubular damage.

* pegylated interferon doesn't cause AKI. REFERENCES

1. Garcia-Tsao G, Parikh CR and Viola A. (2008): Acute kidney injury in cirrhosis. Hepatology. 48(6):2064-2077.

2. Kim DJ, Kang HS, Hyuk Soon Choi HS, et al. (2011): Serum cystatin C level is a useful marker for the evaluation of renal function in patients with cirrhotic ascites and normal serum creatinine levels. The Korean Journal of Hepatology .17(2):130-138.

3. Arroyo V, Terra C and Ginès P. (2007): Advances in the pathogenesis and treatment of type-1 and type-2 hepatorenal syndrome. J Hepatol.46(5): 935-946.

4. Peake M and Whiting M. (2006): Measurement of serum creatinine-current status and future goals. Clin Biochem Rev .27(4):173-184.

5. Sherman DS, Fish DN and Teitelbaum I. (2003): Assessing renal function in cirrhotic patients: problems and pitfalls. Am J Kidney Dis .41(2):269-278.

6. Wong F, Nadim MK, Kellum JA, et al. (2011): Working Party proposal for a revised classification system of renal dysfunction in patients with cirrhosis. Gut . 60(5):702-709 
7. du Cheyron D, Bouchet B, Parienti JJ, et al. (2005): The attributable mortality of acute renal failure in critically ill patients with liver cirrhosis. Intensive Care Med. 31(12):16931699.

8. Mehta RL and Bouchard J. (2011): Controversies in acute kidney injury: effects of fluid overload on outcome Contrib Nephrol. 174 , pp. 200-211

9. Gines P, Angeli P, Lenz K, et al.(2010): EASL clinical practice guidelines on the management of ascites, spontaneous bacterial peritonitis, and hepatorenal syndrome in cirrhosis.J Hepatol. 53(3):397-417.

10. Cholongitas E, Shusang V, Marelli L, et al. (2007): Review article: renal function assessment in cirrhosis-difficulties and alternative measurements. Aliment Pharmacol Ther. 26(7):969-978.

11. Francoz C, Glotz D, Moreau R, et al. (2010): The evaluation of renal function and disease in patients with cirrhosis. J Hepatol . 52(4):605613.

12.Perrone RD, Madias NE and Levey AS. (1992): Serum creatinine as an index of renal function: new insights into old concepts. Clin Chem .38(10):1933 1953

13.Nguyen MT and Devarajan P. (2008):. Biomarkers for the early detection of acute kidney injury. Pediatr Nephrol .23(12):21512157.

14.Caregaro L, Menon F, Angeli P, et al. (1994): Limitations of serum creatinine level and creatinine clearance as filtration markers in cirrhosis. Arch Intern Med .154(2):201-205.

16. Siew ED, Ware LB and Ikizler TA. (2011): Biological markers of acute kidney injury. J Am Soc Nephrol. 22(5):810-820.

16.Singer E, Elger A, Elitok S, et al.(2011): Urinary neutrophil gelatinase-associated lipocalin distinguishes pre-renal from intrinsic renal failure and predicts outcomes. Kidney Int. 80(4): 405-414.

17.Verna E, Farrand E, Pichardo E, et al.(2009): Urinary neutrophil gelatinase associated lipocalin (UNGAL) distinguishes type of kidney failure and predicts mortality in patients with cirrhosis. Hepatology. 50(4):311 A

18. Portal, A.J., McPhail, M.J.W., Bruce, M., et al. (2010): Neutrophil gelatinase-associated lipocalin predicts acute kidney injury in patients undergoing liver transplantation. Liver Transplant . 16(11): 1257-1266.
19. Fagundes C, Pépin M, Guevara M, et al. (2012): Urinary neutrophil gelatinase-associated lipocalin as biomarker in the differential diagnosis of impairment of kidney function in cirrhosis. Journal of Hepatology. 57(2): 267-273.

20. Fraser, A. (2007): Interpretation of liver enzyme tests-A rapid guide. New Zealand Family Physician (NZFP). 34 (3):194-196.

21.Gungor, G., Ataseven, H., Demir, A., et al. (2014): Neutrophil gelatinase-associated lipocalin in prediction of mortality in patients with hepatorenal syndrome: a prospective observational study. Liver International . 34(1):49-57.

22. Montalbano, M., Pasulo, L., Sonzogni, A., et al (2007): Treatment with pegylated interferon and ribavirin for hepatitis $\mathrm{C}$ virus-associated severe cryoglobulinemia in a liver/kidney transplant recipient. J Clin Gastroenterol. 41(2):216-220.

23. Wittho Th, Moller, B., Wiedmann, K.H., et al. (2007): Safety, tolerability and efficacy of peginterferon alpha-2a and ribavirin in chronic hepatitis $\mathrm{C}$ in clinical practice: The German Open Safety Trial. Journal of Viral Hepatitis. 14(11): 788-796.

24. Kamal, S., Madwar, M., Bianchi, L., et al. (2000): Clinical, virological and histopathological features: long-term follow-up in patients with chronic hepatitis C co-infected with S. mansoni. Liver. 20(4):281- 289.

25.Mahmoud, K.M., Sobh, M.A., El Agroudy, A.E., et al. (2001): Impact of schitosomiasis on patient and graft outcome after renal transplantion: 10 years follow up.Nephrol Dial Transplant.16(11):2214-2221.

26. Abbas, O.M., Omar, N.A., Zaghla, H.E., et al. (2009): Schistosoma mansoni coinfection could have a protective effect against mixed cryoglobulinaemia in hepatitis $\mathrm{C}$ patients. Liver international. 29 (7): 1065-1070.

27.Verna, E.C., Brown, R.S., Farrand, E., et al. (2012): Urinary Neutrophil GelatinaseAssociated Lipocalin Predicts Mortality and Identifies Acute Kidney Injury in Cirrhosis. Dig Dis Sci . 57(9):2362-2370.

28.Ochs, A., Rossle, M., Haag, K., et al. (1995): The transjugular intrahepatic portosystemic stent-shunt procedure for refractory ascites. N Engl J Med. 332(18):1192-1197.

29.Cassinello, C., Moreno, E., Gozalo, A., et al. (2003): Effects of orthotopic liver transplantation on vasoactive systems and renal function in 
patients with advanced liver cirrhosis. Dig Dis Sci. .48(1):179-186.

30.McDonald, F.D., Brennan, L.A and Turcotte, J.G. (1973): Severe hypertension and elevated plasma renin activity following transplantation of "hepatorenal donor" kidneys into anephric recipients. Am J Med. 54(1):39-43.

31.Kanel, G.C and Peters, R.L. (1984): Glomerular tubular reflux-a morphologic renal lesion associated with the hepatorenal syndrome. Hepatology. 4(2):242-246.

32.Hollenberg, N.K. (2004): Aldosterone in the development and progression of renal injury. Kidney Int. 66(1):1-9.

23.Niculae,A., Checherita, I and Ciocalteu,A.(2013): Is NGAL useful in prediction of hepatorenal syndrome diagnosis in patients with hepatic cirrhosis? International Society Of Nephrology.World congress of nephrology. abstracts. Session Info: Moderated Poster Session: Biomarkers for Acute Kidney Injury.Suppl:143

34.El Bassat, H., Ziadaa, D.H., Taha, A., et al. (2014): Urinary neutrophil gelatinase-associated lipocalin as a biomarker for the diagnosis of hepatorenal syndrome in cirrhotic patients. Tanta Medical Journal 41(4)-346-352.

35.Mori K and Nakao K. (2007): Neutrophil gelatinase-associated lipocalin as the real-time indicator of active kidney damage. Kidney Int. 71(10):967-970. 